Article

\title{
Effects of Double Reduction and Annealing on the Texture during Continuous Rolling of Ultrathin Sheets of Low Carbon Steels
}

\author{
Laura G. Castruita-Avila 1,* , Francisco A. García-Pastor 1, Manuel de J. Castro-Roman 1, \\ Jesus Emilio Camporredondo-Saucedo ${ }^{2}$, Fabián Equihua-Guillen ${ }^{2}$ and Jimy Unfried-Silgado ${ }^{3}$ \\ 1 CINVESTAV-IPN Unidad Saltillo, Industria Metalúrgica 1062, Parque Industria Saltillo, Ramos Arizpe, \\ Ramos Arizpe 25900, México; E-mails : f.g.pastor@microestructura.org (FAGP) ; 4mcastro@gmail.com \\ (MJCR) \\ 2 Facultad de Ingeniería Mecánica y Eléctrica, Universidad Autónoma de Coahuila; Av. Barranquilla S/N, Col \\ Guadalupe, Monclova 25750, México. E-mails: camporredondo05@gmail.com (EC); \\ fabianequihua@gmail.com (FE) \\ 3 Departamento de Ingeniería Mecánica. Grupo ICT. Universidad de Córdoba. Carrera 6 No. 76-103, ZIP \\ 230002 Montería, Colombia; e-mail: jimyunfried@correo.unicordoba.edu.co \\ * Correspondence author: castruita_laura@hotmail.com; Tel.: +52-844-438-9600
}

\begin{abstract}
In this paper were analyzed the effects of double reduction and annealing during rolling process on texture evolution in an ultrathin sheet of low carbon steel. Experimental samples were obtained from each process stage. EBSD technique and correlated tools as orientation density functions and pole figures were used to analyze the microstructural changes and the texture. Results show that $\{111\}$ recrystallized grains were formed during process, reducing dramatically $\gamma$-fibre texture intensity and generating an adequate finished product for deep die stamping.
\end{abstract}

Keywords: Cold Rolling, Texture, EBSD, ultrathin sheet.

\section{Introduction}

Nowadays there is a great interesting by manufacturing of ultrathin steel sheets with outstanding mechanical properties. Aiming to obtain smaller thicknesses, it is necessary to induce large plastic deformations that produce both microstructure and texture changes and affecting its formability. On these conditions is unlikely that the plate can be conformed as finished product. The annealing could be used aiming to release residual stresses and improved the behavior of the microstructure. Due to these changes could be advisable to analyze the relationships among the deformation and texture during the different stages of the process. At this point, it is fundamental to analyze the microstructural phenomena that occur during continuous annealing, such as, recovery, recrystallization and grain growth aiming to improve the mechanical properties during shaped [1-2].

Consequently, after applied high strain to plates during rolling are expected a deformed grain microstructure and increasing of internal defects. Subsequently, during the first stage of continuous annealing, the rearrangement and the reduction in dislocations density of the deformed structure starts. This process is called recovery. In the next step, known as recrystallization, a new grain structure is formed from existed nuclei, promoting an equiaxed grain structure. Intensity of recrystallization on microstructure depends on available driving force (internal energy generated from deformation), temperature, and stacking fault energy (SFE), which in turn depends of type lattice crystal. The grain growth occurs from subgrain groups which reach the adequate both size and the misorientations to grow and form a grain. It is possible to occur abnormal growth of grain, especially if external conditions that generates recrystallization are keeping. From of viewpoint of metallurgical behavior, the properties of a polycrystalline aggregates depend on individual grains. Grains could be randomly oriented or to show a tendency to one or several crystallographic orientations. The sum of the crystallographic orientations of the crystallites in a polycrystalline 
aggregate is known as the texture [2]. Then, Texture is the distribution of crystallographic orientations with respect to a sample reference frame, which affects the grain-to-grain misorientations. The evolution of the microstructure and texture in low carbon steels processed by plastic deformation are produced by a variation in its energy stored during the deformation. In the case of highly deformed steels, stored energy is mainly concentrated as generation and interaction between dislocations [3-16]. Abovementioned applies too for continuous annealing process where both mechanical properties and microstructure are altered [7-12].

Commonly, texture analysis is conducted by Electron Back Scattering Diffraction (EBSD) technique coupled to scanning electron microscopy SEM, which provides information of diverse aspects such as the texture components, the grain-to-grain misorientations, the grain size, and as well as, phase identification of the material. EBSD technique has advantages such as quickly application and reproducibility. Texture measurements using EBSD techniques provides results by means of Orientation Distribution Functions (ODFs), usually representing the frequency distribution of the continuum of orientations in Euler Space. $[5-6,15,18]$. ODFs are used to determine the texture in main components of the angles, $\varphi, \varphi_{1}, \varphi_{2}$ represented by a tridimensional view of the Euler space within the Bunge context [8-9].

Low carbon steels are a group of well-known Fe-C alloys with predominantly BCC ferritic microstructure, which attains its final mechanical properties through thermomechanical treatments. In BCC crystals the most important fibre of texture in $\varphi_{2}=45^{\circ}$ are $\gamma$-fibre $<111>/ / \mathrm{ND}$ with main components in (111)[1 $\overline{2} 1]$, (111)[0 $\overline{1} 1],(111)[\overline{1} \overline{1} 2], \alpha$-fibre $<110>/ / R D$ with main components in

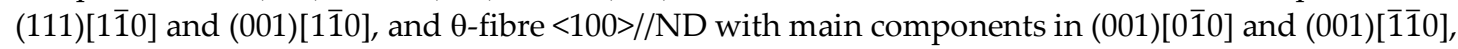
[19]. In the case of ultrathin sheets of low carbon steels processed by double reduction, the continuous annealing treatments could be highly effective because the sheet deformation process occurs in relatively short time. A strict control during the ultrathin sheet fabrication stages could result in texture that benefits properties of finished products. However, the effect of a double reduction and subsequent annealing on the final texture has not been properly studied yet. Therefore, the goal of this work is to study the texture evolution during the double reduction cold rolling process with application of continuous annealing aiming to suggest process improvements without affecting the finished product quality.

\section{Materials and Methods}

\subsection{Cold Rolling Process}

Low carbon ferritic steel sheet with an initial thickness of $2 \mathrm{~mm}$, which was obtained from hot rolling process has been used as experimental material. The deformation process starts with the first cold reduction, achieving a deformation of $76 \%$. Subsequently, the thinplate was processed in a continuous annealing line heating the sheet up to $690^{\circ} \mathrm{C}$. In order to obtain a final thickness of 0.22 $\mathrm{mm}$, the second cold reduction was about $53 \%$. Finally, the finished product was obtained in a mill which provides a light mechanical tempering with an elongation in the range of $0.6-1.0 \%$. Table 1 details the low carbon steel production sequence as well as the variation of the deformed sheet thickness due to the cold rolling. In Figure 1 is shown complete information about of cold rolling process and extraction of experimental samples.

Table 1. Thickness variation of thinplate during the reduction process $(\mathrm{mm})$.

\begin{tabular}{ccccccc}
\hline $\begin{array}{c}\text { Raw } \\
\text { Coil }\end{array}$ & $\begin{array}{c}\text { First } \\
\text { Reduction }\end{array}$ & $\begin{array}{c}\text { First } \\
\text { Annealing }\end{array}$ & $\begin{array}{c}\text { Second } \\
\text { Reduction }\end{array}$ & $\begin{array}{c}\text { Second } \\
\text { Annealing }\end{array}$ & $\begin{array}{c}\text { Tempered } \\
\text { Steel }\end{array}$ & $\begin{array}{c}\text { Finished } \\
\text { Product }\end{array}$ \\
\hline 2.0 & 0.46 & 0.46 & 0.22 & 0.22 & 0.22 & 0.22 \\
\hline
\end{tabular}




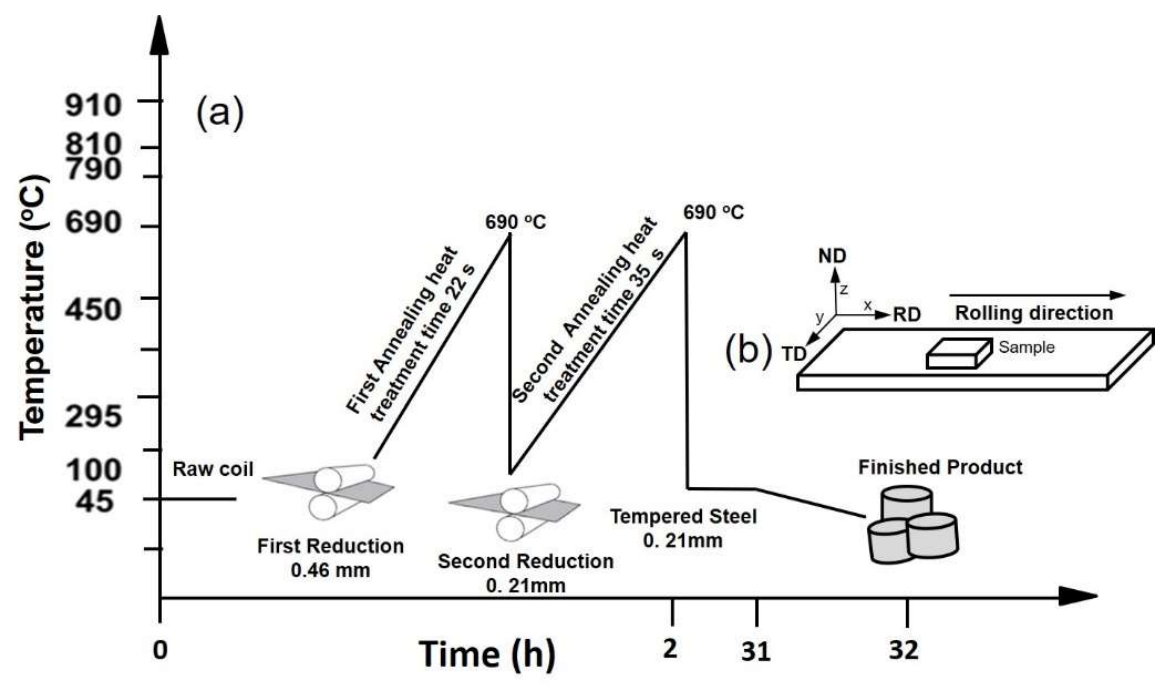

Figure 1. (a) Sequence of cold rolling process shows time and temperatures. (b) Symmetry references of experimental samples.

2.2 Chemical Composition

Chemical analysis of the samples were obtained by optical emission spectroscopy and its results are shown in Table 2.

Table 2. Chemical composition of the experimental steel (Mass \%).

\begin{tabular}{cccccccccccc}
\hline $\mathbf{C}$ & $\mathbf{S i}$ & $\mathbf{M n}$ & $\mathbf{P}$ & $\mathbf{S}$ & $\mathbf{C r}$ & $\mathbf{M o}$ & $\mathbf{N i}$ & $\mathbf{A l}$ & $\mathbf{C u}$ & $\mathbf{T i}$ & $\mathbf{V}$ \\
\hline 0.04 & 0.008 & 0.154 & 0.029 & 0.015 & 0.022 & 0.003 & 0.024 & 0.020 & 0.032 & 0.001 & 0.003 \\
\hline
\end{tabular}

\subsection{Sample metallographic preparation}

$1 \times 1 \mathrm{~cm}^{2}$ samples were cut from rolling processed plates, having special careful with traceability during the process with rolling directions. Samples were grounded on progressively finer $\mathrm{SiC}$ abrasive papers up to granulometry of 2400 . The samples were polished until a metallographic quality specular finished, using microfiber clothes and diamond paste of 0.3 and 0.1 $\mu \mathrm{m}$. The cleaning process was made using a STRUERS® ultrasonic machine. Final polishing for EBSD analysis was performed using colloidal silicon suspension with particle size of $0.4 \mu \mathrm{m}$. Samples for metallography characterization were etched in a Nital 3\% solution for 15 seconds.

\subsection{Microstructure and Texture Characterization}

Microstructure and texture characterization were carried out using a scanning electron microscope PHILIPS XL 30 ESEM ${ }^{\circledR}$ coupled to TSL OIM Analysis TM EDAX software. References of symmetry of each sample were located similarly those related to rolling directions, i.e. $\mathrm{x}$-axis was parallel to rolling direction $\mathrm{RD}, \mathrm{y}$-axis in the transversal direction $\mathrm{TD}$, and z-axis in the normal direction $\mathrm{ND}$, respectively (Figure 1b). It was applied the technique of Orientation Imaging Microscopy (OIM) in each analyzed sample, for which were analyzed and obtained both pole figures and ODF's. An area of $150 \mu \mathrm{m} \times 200 \mu \mathrm{m}$ was scanned using a spatial resolution of $1 \mathrm{~nm}$ to $15 \mathrm{kV}$. The symmetry of the samples was fixed, as orthotropic body, using an iteration method by means of harmonic series expansion in the range of $\mathrm{L}=16$ and Gaussian softening of $5^{\circ}$.

\section{Results and Discussion}

The microstructure and the texture of the low carbon steel here studied have undergone important changes during the cold rolling and the successive continuous annealing processes. OIM 
results for each stage of deformation processing are shown in Figure 2. The analysis of OIM maps images show that the texture material presents difference in misorientations and grain size along of conformation process. Equiaxed grains are observed in the following stages: first and second annealing, tempered and finished, besides raw coil state. Meanwhile, first and second reduction show elongated grains (more evident in first reduction how it was expecting), exhibiting a tendency to RD direction alinement. After there were applied both first and second annealing were observed recrystallized grains in some regions.

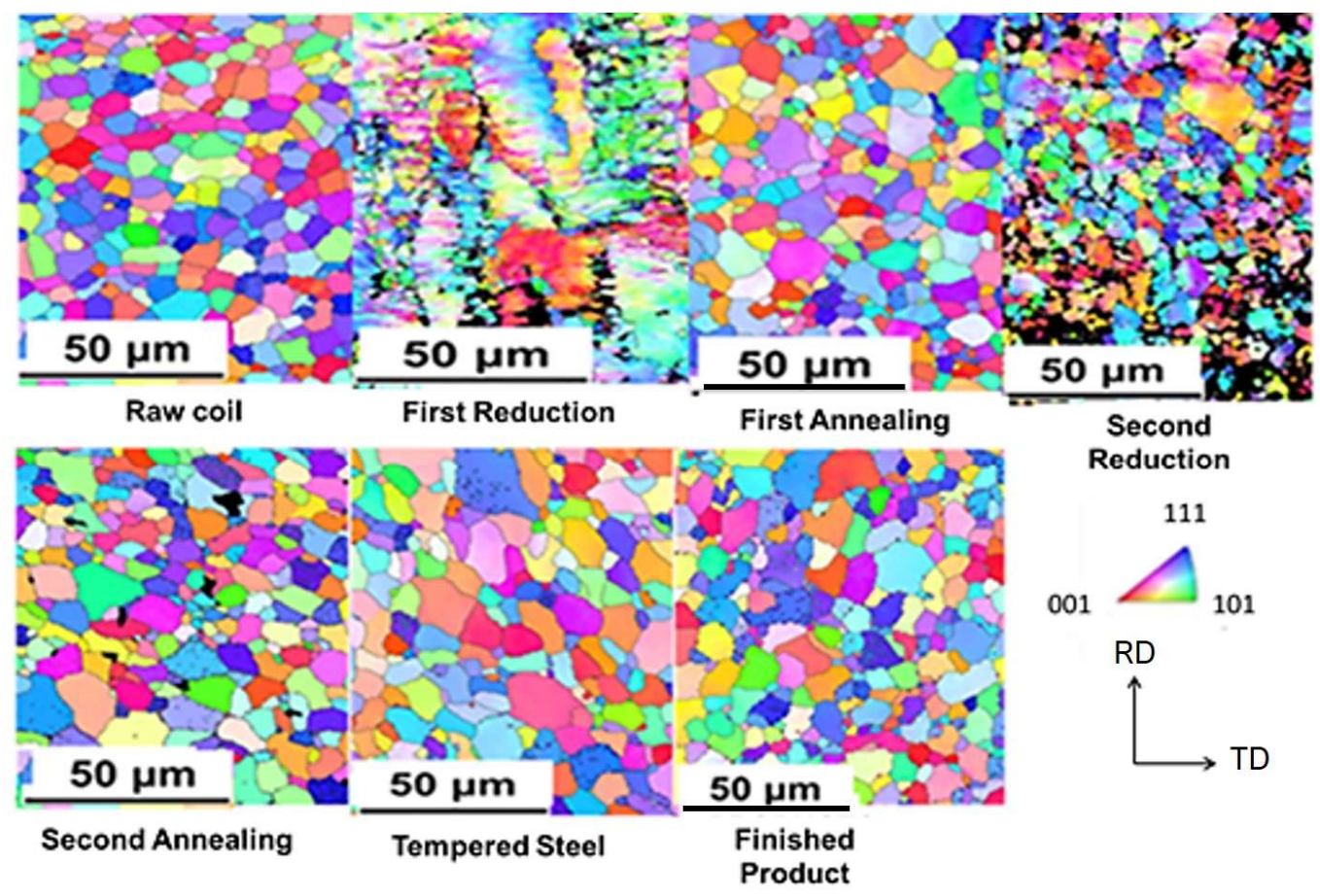

Figure 2. Images of the EBSD maps obtained from each stage during cold rolling process.

In the Figure 3 are shown the textures through (111) pole figures (PF's) for each stage of applied rolling process. It can be observed that in first and second stages of reduction the PF's show a greater intensity in the preferential texture formation in the component (111), pointing out that the most important fibre texture in this process is $\gamma$-fibre [19]. Annealing textures (first and second) show that intensity in component (111) continous being strong, suggestioning that recristallization nuclei begins from deformed regions at the same preferential direction (111) [20]. Lately, the preferential texture fibre is preserved at the stages of tempered and finished of product. 


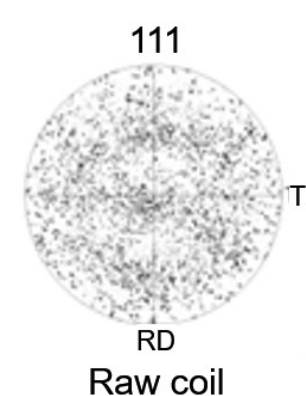

Raw coil

111

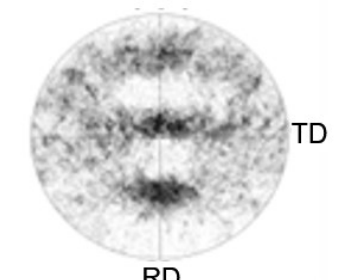

$\mathrm{RD}$

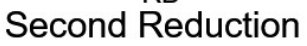

111

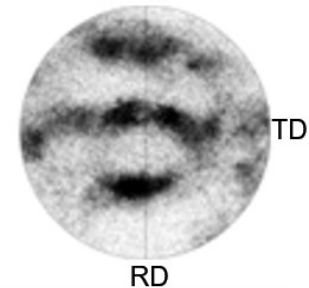

First Reduction

111

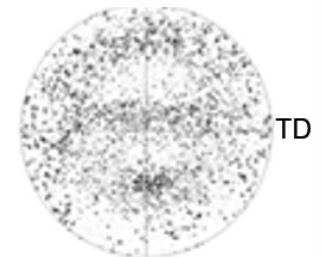

RD
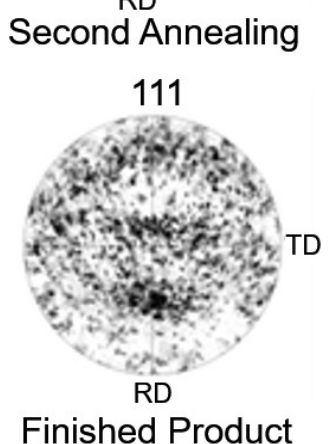

Finished Product

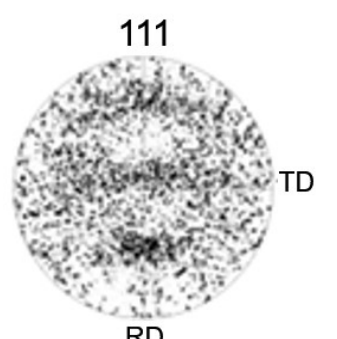

$\mathrm{RD}$

First Annealing

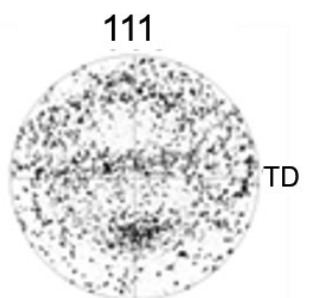

$R D$

Tempered Steel

Figure 3. (111) Pole figure showing textures for each stage of rolling process.

In the case of ferritic steels such as those analyzed in this work, used for deep die-stamping, usually it has been found texture (called as $<111>/ / N D$ ), in planes $\{111\}$ parallel to the normal direction well-known as $\gamma$-fibre. In this texture is feasible to observer that the grains have high stored energy, while the grains with the direction $<110>$ parallel to the rolling direction belong to the $\alpha$-fiber (called as $<110>/ / R D$ ) contain a lower energy. It has been observed that as the amount of $\gamma$-fibre has an inverse relationship with the amount of $\alpha$-fibre [11-13].

Figure 4 shows ODF's results with $\varphi_{2}=45^{\circ}$ for each stage of process. The ODF corresponding to raw coil (Figure 4a) shows the highest density in the components (001)[1]10] on RD $\theta$-fibre and (111)[110] and (111)[ $\overline{1} \overline{1} 2]$ on ND $\gamma$-fibre, respectively. When the sample is subjected to the first reduction $(76 \%)$, appears a preferential texture on ND $\gamma$-fibre in component (111)[ $1 \overline{1} 2$ ], which is an essential characteristic of steels with good formability [10-17]. During the first annealing, the greatest intensity was registered in component $(001)[\overline{1} \overline{1} 0]$ on RD $\theta$-fibre validating the grain rotation around $<111>/ / \mathrm{ND}$ [19].

ODF's of second reduction shows preference by the component (554)[ $\overline{2} \overline{2} 5]$ on ND $\gamma$-fibre, a typical texture observed in deformed low carbon steels [19,20]. After second annealing, texture

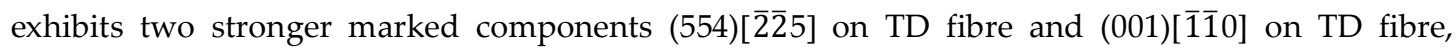
confirming once more, grain rotation around $<111>/ / \mathrm{ND}$. Tempered stage of the steel shows a strong

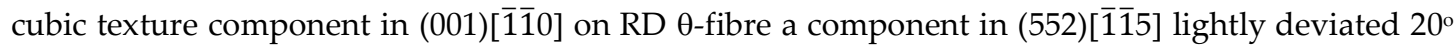


6 of 10

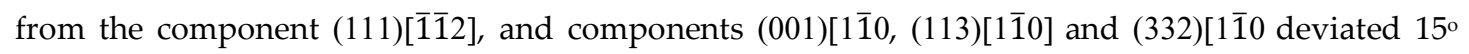
from the component (111)[11 10 .

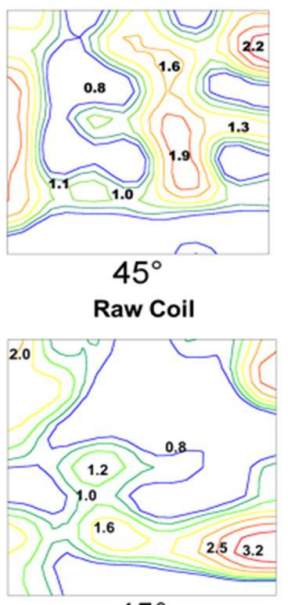

$45^{\circ}$
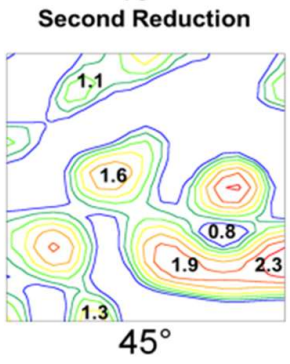

Finished Product

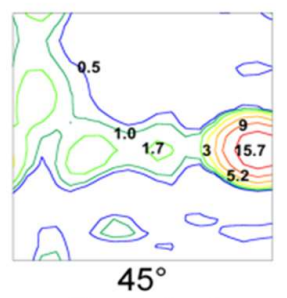

First Reduction

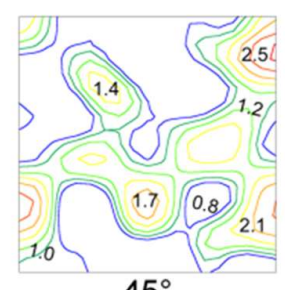

$45^{\circ}$

Second Annealing

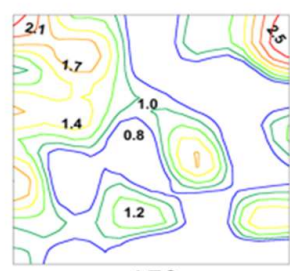

$45^{\circ}$

First Annealing

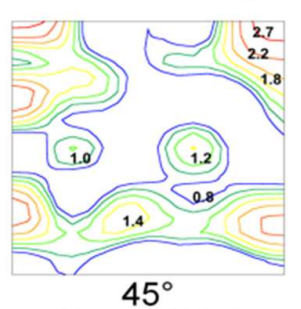

Tempered Steel

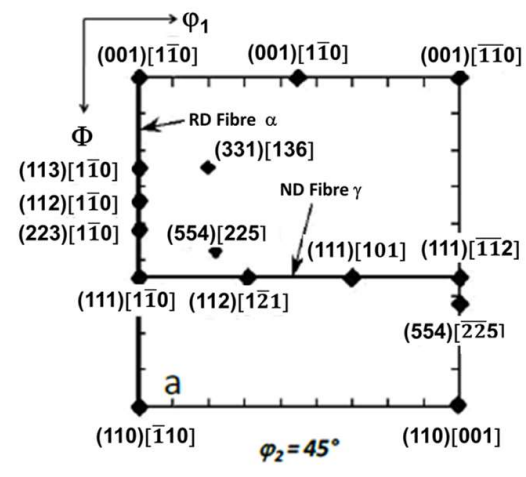

Constant Angle: $\varphi 2$

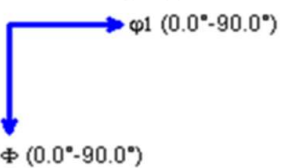

Texture Name: Harmonic:L=16, HW=5.0

Calculation Method: Harmonic Series Expansion

Series Rank (I): 16

Gaussian Smoothing: $5.0^{\circ}$

Sample Symmetry. Orthotropic

Representation: Euler Angles (Bunge)

Figure 4. ODF's maps within the Bunge context at $\varphi 2=45^{\circ}$ during the cold rolling process.

Finally, finished product shows stronger marked component (554)[ $\overline{2} \overline{2} 5]$ on TD around $\gamma$-fibre grains [21], additionally, marked components in (554)[225], in (221)[1 $\overline{1} 0]$ deflected 25\% from

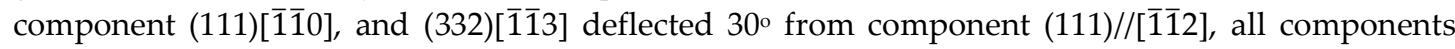
with rotations around $<111>/ / \mathrm{ND}$. In accordance to results abovementioned ultrathin low carbon steel sheet has been completely recrystallized. Tempered steel sheet underwent a deformation of 0.6 $-1 \%$ which negatively affects the fiber $\gamma$ intensity, demonstrating that the final texture depends on the magnitude of the deformation.

In Figure 5 are shown the $\gamma$-fibre intensity for each one of rolling process stages. Results shows that intensity of $\gamma$-fibre in first reduction stage is higher than remainder of process stages. Furthermore, finished product stage is the second stage with higher intensity of $\gamma$-fibre. This behavior is common in highly deformed steels [8]. 


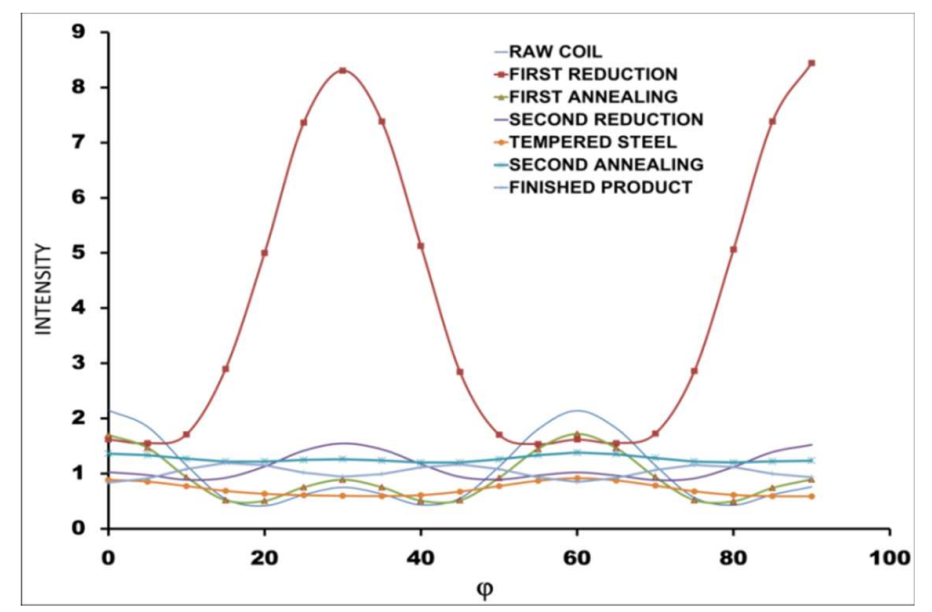

Figure 5. $\gamma$-Fibre intensity rates obtained during rolling process stages.

Figure 6 shows the evolution of misorientation angles during rolling process stages. Severe deformation, such as both the first and second reductions, has shown misorientations angles $\leq 15^{\circ}$, which is coherent with substructure of dislocations into deformed grains. Recrystallized samples from first and second annealing, just as finished product stage showed misorientations angles $\geq 30^{\circ}$ with randomized values, demonstrating that material has been completely recrystallized.
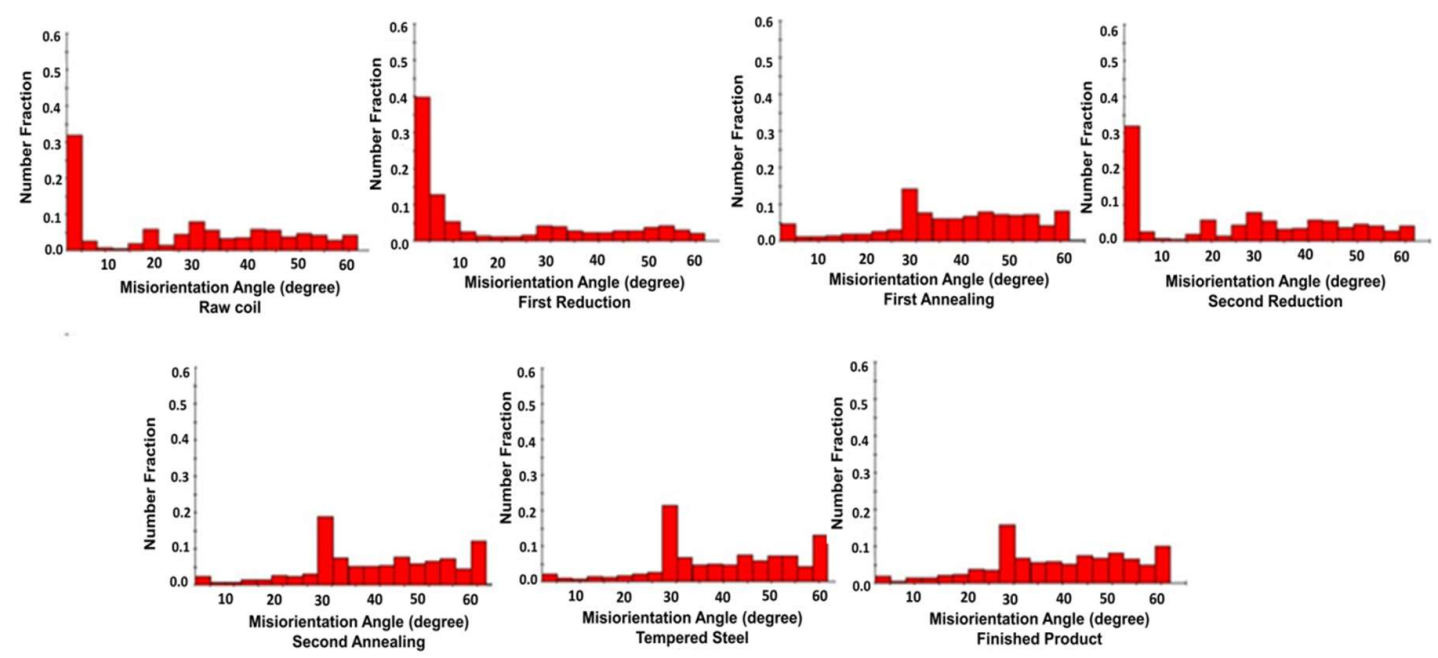

Figure 6. Histograms of the disorientation angle evolution during the cold rolling process.

\section{Conclusions}

Significant changes in the evolution of texture have been observed when was applied a double reduction and double annealing during continuous rolling process aiming to obtain a $0.22 \mathrm{~mm}$ ultrathin sheet from a low carbon steel. Texture analysis showed that mean components after application of complete rolling process showing dramatically reduction of $\gamma$-fibre texture intensity with a completely recrystallized rotated grains around $\{111\}$ directions. Finished product showed a marked component (554)[ $\overline{2} \overline{2} 5]$ on TD around $\gamma$-fibre grains.

Acknowledgments: This work was realized thanks to the financial support from CONACYT, facilities provided by CINVESTAV, Saltillo Unit and the AHMSA group who provided the material required in this study.

Author Contributions: LGCA, FAGP and MJCR conceived and design the experiments, LGCA performed the experiments, analyzed data and wrote the draft of manuscript. All authors, including JECS, JUS and FEG took part in the discussion, the preparation and revision of the manuscript. 
Conflicts of Interest: The authors declare no conflict of interest. The founding sponsors had no role in the design of the study; in the collection, analyses, or interpretation of data; in the writing of the manuscript, and in the decision to publish the results.

\section{Abbreviations}

The following abbreviations are used in this manuscript:

EBSD: Electron Back Scattering Diffraction

OIM: $\quad$ Orientation Imaging Microscopy

IQ: $\quad$ Image Quality

ODF's: Orientation Density Functions

\section{References}

1. S. Suwas, R. Kumar-Ray. Crystallographic Texture of Materials. Springer-Verlag London 2014:143-178.

2. F.J. Humphreys, M. Hatherly. Recrystallization and related Annealing Phenomena. Elsevier, Oxford, 1st ed. 1995:3.

3. M. J. Godec. Presentation methods of textures measurements. Materiali in Tehnoloije. 2000 (34):359-364.

4. R. D. Doherty, D.A. Hughes. et al. Current issues in recrystallization: a review, Materials Science Engineering. 1997: 219-274.

5. T. Watanabe, S. Tsurekawa. et al. The Coming of Grain Boundary Engineering in the 21st Century. Microstructure and Texture in Steels, (c) Springer. 2009: 43-82.

6. S. Choi, Y. Sool-Jin. Evaluation of stored energy in cold-rolled steels from EBSD data. Materials Science and Engineering. 2004:149-159.

7. J. Hamada, N. Ono. Effect of Microstructure before Cold Rolling on Texture and Formability of Duplex Stainless Steel Sheet. Materials Transactions. 2010 (51)(4):635- 643

8. B. Hutchinson, Deformation microstructures and textures in steels Phil. Trans. R. Soc. Lond. 357, doi: 10.1098/rsta. Published 1999:1471-1485.

9. J. Jonas. Transformation textures associated with steel processing. Microstructure and Texture in Steels and Other Materials. Proceedings of the international. Conference on microstructure and texture in steels and other materials. Jamshedpur, India 2008:3-16.

10. T. He, Y.D. Liu. et al. Study on the Micro Mechanism of Recrystallization Texture Formation in Cold-rolled IF Steel sheet. Materials Science Forum. 2005 (495-497):417-422.

11. D. Raabe, K. Lucke, Rolling and annealing textures of BCC Metals Materials Science Forum. Trans Tech Publications, Switzerland. 1994:157-162

12. Hai-Tao Liu, J. Schneider. et al. Microstructure and Texture Evolution in Non-oriented Electrical Steels Along Novel Strip Casting Route and Conventional Route. Steel Research int. 86. 2015.

13. P. Gobernado, R. Petrov. et. al. Texture Evolution in Si-Alloyed Ultra Low-Carbon Steels after Severe Plastic Deformation. Advanced Engineering Materials. 2010 (10): 1077-1081.

14. Olaf Engler, Valerie Randle. Introduction to Texture Analysis, Macrotexture, Microtexture, and Orientation mapping. CRC Press Taylor d Francis Group. 2010.

15. Zhongwei Chen, Yanqing Yang. et al. Some Applications of Electron Back Scattering Diffraction (EBSD) in Materials Research. Scanning Electron Microscopy Edited by Dr. Viacheslav Kazmiruk. 2012:55-72.

16. D. Jr. Callister. Fundamentals of materials science and engineering / an Interactive. Augusto 2000.

17. E. Novillo, M. Petite. et al. Texture and Microstructure Evolution in an ultra-low carbon steel during recristallization. Advanced Engineering Materials. 2003 (8):575-578.

18. J. Schwartz, M. Kumar et al. Electron Backscatter Diffraction in Materials Science. Springer Science. Second Edition. 2009:1.

19. L. A. I. Kestens \& H. Pirgazi. Texture formation in metal alloys with cubic crystal structures, Materials Science and Technology. 20016 (32:13):1303-1315.

20. H. Inagakl. Fundamental Aspect of Texture Formation in Low Carbon Steel, Review SIJ International. 1994 (34- 4): 313-321

21. AI Nosenkov, et al. Effect of alloying elements on the microstructure and texture of warm rolled steels."ISIJ international 44.4. 2004: 717-724. 